\title{
Mean field bipartite spin models treated with mechanical techniques
}

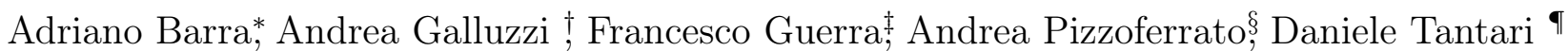

September 11, 2018

\begin{abstract}
Inspired by a continuously increasing interest in modeling and framing complex systems in a thermodynamic rationale, in this paper we continue our investigation in adapting well known techniques (originally stemmed in fields of physics and mathematics far from the present) for solving for the free energy of mean field spin models in a statistical mechanics scenario.

Focusing on the test cases of bipartite spin systems embedded with all the possible interactions (self and reciprocal), we show that both the fully interacting bipartite ferromagnet as well as the spin glass counterpart, at least at the replica symmetric level, can be solved via the fundamental theorem of calculus, trough an analogy with the Hamilton-Jacobi theory and lastly with a mapping to a Fourier diffusion problem. All these technologies are shown symmetrically for ferromagnets and spin-glasses in full details and contribute as powerful tools in the investigation of complex systems.
\end{abstract}

\section{Introduction}

In the last years, equilibrium statistical mechanics has been successfully extended beyond the conventional area of the physics of matter, for instance in quantitative sociology (see e.g. [13, 25, 20, 21]) or theoretical biology (see e.g. [38, 3, 37, 4]). However, these (as well as many others, see e.g. [16, 23]) new research fields continuously require more refined mathematical methods and models in order to give an always more relevant quantitative description and understanding of the phenomena they aim to tackle.

Among the several novelty these fields of research required, there has been a microscopic description of dynamical systems where two species compete or collaborate, for instance a' la Lotka-Volterra: restricting to equilibrium properties, this need led to a renewal formulation of bipartite spin systems 22], beyond their original introduction within the more standard world of physics of matter [32], which allows to study the emergent collective properties of two interactive large groups of variables. For instance, in quantitative sociology the latter may capture essential features of migrant's integration inside a host community [14] or the dialogue between two different ensembles of closely interacting cells, as for instance B and T cells within the immune system [1].

In this paper we do not deal with comparing modeling to real data, instead we continue our investigation consisting in obtaining new statistical mechanics techniques all based on adapting existing technologies originally developed to work in field far away from the actual focus, such to make them able to solve for the free energy of suitably defined mean field spin Hamiltonians. We will mainly focus on sum rules originated from a mapping of the statistical mechanics problem with the fundamental theorem of calculus as firstly shown in [30] and then extended in [9], with the Hamilton-Jacobi framework, firstly developed in [28] and then extended in [9] 12], and with the Fourier conduction investigated in [26] 7, which is a side effect of the mechanical analogy previously introduced.

Dealing with the subjects and not only with the methodologies, the present work constitutes an extension mainly of [6], where bipartite mean field model have been carefully inspected from a (standard) statistical mechanics

\footnotetext{
*Sapienza Università di Roma, Dipartimento di Fisica and GNFM Gruppo di Roma, Italy

†Sapienza Università di Roma, Dipartimento di Matematica and GNFM Gruppo di Roma, Italy

${ }^{\ddagger}$ Sapienza Università di Roma, Dipartimento di Fisica and INFN Sezione di Roma, Italy

$\S$ The University of Warwick, Mathematics Institute, Coventry, United Kingdom

`Sapienza Università di Roma, Dipartimento di Matematica and GNFM Gruppo di Roma, Italy
} 
perspective and [7] where the techniques we are going to use have been tested on single-party models: the two routes of investigation are here merged together in a unified and stronger theory.

The paper is divided into two symmetric parts: In the first one, a bipartite ferromagnetic model, which not only considers the interaction among spins of different parties but also between the ones of the same group, is studied through three different interpolation approaches, respectively the fundamental theorem of calculus, the Hamilton-Jacobi scheme and the Fourier transform. In the second one, the same procedures are applied to the disordered (glassy) counterpart of the first model. Unfortunately, as a Parisi-like theory [36] for these models is still under construction, and also because it is usually sacrificed in many practical applications involving models beyond the Sherrington-Kirkpatrick paradigm, the thermodynamics of these systems is studied at the replica symmetric level.

\section{Ferromagnetic case}

\subsection{The Model}

The spin system we study is an extension of the one analyzed in [6]. There, two dichotomic parties of variables, $\left\{\sigma_{i}\right\}_{i=1, \ldots, N_{\sigma}}$ and $\left\{\tau_{i}\right\}_{i=1, \ldots, N_{\tau}}$, which were coupled through a ferromagnetic interaction, were considered: here we take into account also the ferromagnetic interaction between spins of the same group. All this results in a Hamiltonian made up of the following contributions

$$
H_{N}(\boldsymbol{\sigma}, \boldsymbol{\tau}, \boldsymbol{\beta})=-\frac{1}{N} \beta_{\sigma \tau} \sum_{i=1}^{N_{\sigma}} \sum_{j=1}^{N_{\tau}} \sigma_{i} \tau_{j}-\frac{1}{2 N} \beta_{\sigma} \sum_{i, j}^{N_{\sigma}} \sigma_{i} \sigma_{j}-\frac{1}{2 N} \beta_{\tau} \sum_{i, j}^{N_{\tau}} \tau_{i} \tau_{j},
$$

where $\sigma_{i}, \tau_{i} \in\{-1 ; 1\}$ are the two families of dichotomic spin variables; $\beta_{\sigma}, \beta_{\tau}$ and $\beta_{\sigma \tau}$ are the strength of the interactions weighting the intensity of the three different contributions to the Hamiltonian; $N_{\sigma}$ and $N_{\tau}$ are the number of spins for each party with $N=N_{\sigma}+N_{\tau}$. Note that equation (1) defines a mean-field model, where each couple of spins interact in a ferromagnetic way (all the couplings are positive), and the normalization $1 / N$ ensure the linear extensivity of the thermodynamical observables (e.g. energy, entropy, etc.) with respect to the size of the system. Introducing $\alpha=N_{\sigma} / N$ and thus $(1-\alpha)=N_{\tau} / N$ and denoting with $\mathcal{O}(\boldsymbol{\sigma}, \boldsymbol{\tau})$ a generic observable of the system, the definitions of the statistical mechanic and thermodynamic quantities are given straightforwardly:

\section{Partition function}

Boltzmann average

Magnetization of the $\sigma$ party

Magnetization of the $\tau$ party

Pressure (free energy)

$$
\begin{gathered}
Z_{N}(\boldsymbol{\beta}, \alpha):=\sum_{\boldsymbol{\sigma}, \boldsymbol{\tau}} e^{-\beta H_{N}(\boldsymbol{\sigma}, \boldsymbol{\tau}, \boldsymbol{\beta}, \alpha)}, \\
\langle\mathcal{O}(\boldsymbol{\sigma}, \boldsymbol{\tau})\rangle:=Z_{N}^{-1}(\boldsymbol{\beta}, \alpha) \sum_{\boldsymbol{\sigma}, \boldsymbol{\tau}} \mathcal{O}(\boldsymbol{\sigma}, \boldsymbol{\tau}) e^{-\beta H_{N}(\boldsymbol{\sigma}, \boldsymbol{\tau}, \boldsymbol{\beta}, \alpha)}, \\
m_{\sigma}(\boldsymbol{\sigma}):=\frac{1}{N_{\sigma}} \sum_{i=1}^{N_{\sigma}} \sigma_{i}, \\
m_{\tau}(\boldsymbol{\tau}):=\frac{1}{N_{\tau}} \sum_{i=1}^{N_{\tau}} \tau_{i}, \\
A(\boldsymbol{\beta}, \alpha)=\lim _{N \rightarrow \infty} A_{N}(\boldsymbol{\beta}, \alpha):=\frac{1}{N} \ln Z_{N}(\boldsymbol{\beta}, \alpha)=-\beta f_{N}(\beta),
\end{gathered}
$$

where $f_{N}(\beta)$ is the free energy.

In the following, for the sake of simplicity and without loss of generality, we will put $\beta=1$ : we can restore the dependence by $\beta$ simply rescaling the couplings $\beta_{x} \rightarrow \beta \beta_{x}$, with $x=\sigma, \tau, \sigma \tau$. In the present paper we want to describe three different techniques that can be used to solve the model and in particular to compute the thermodynamic limit of the intensive pressure as to characterize the thermodynamic states, i.e. the averages (and in general the moments) of the order parameters. Each one of the three routes approaches the problem from a different perspective but all of these can be thought as proofs of the following 
Theorem 1. The thermodynamic limit of the intensive pressure of the full interacting ferromagnetic bipartite model defined in (1) reads as

$$
\begin{aligned}
A(\boldsymbol{\beta}, \alpha) & =\ln 2+\alpha \ln \cosh \left(\beta_{\sigma} \alpha \bar{m}_{\sigma}+\beta_{\sigma \tau}(1-\alpha) \bar{m}_{\tau}\right)+(1-\alpha) \ln \cosh \left(\beta_{\sigma \tau} \alpha \bar{m}_{\sigma}+\beta_{\tau}(1-\alpha) \bar{m}_{\tau}\right)+ \\
& -\left[\beta_{\sigma \tau} \alpha(1-\alpha) \bar{m}_{\sigma} \bar{m}_{\tau}+\frac{1}{2} \beta_{\sigma} \alpha^{2} \bar{m}_{\sigma}^{2}+\frac{1}{2} \beta_{\tau}(1-\alpha)^{2} \bar{m}_{\tau}^{2}\right],
\end{aligned}
$$

where the two quantities $\bar{m}_{\sigma}$ and $\bar{m}_{\tau}$ are the solution of the following system of self-consistent equations

$$
\left\{\begin{array}{l}
\bar{m}_{\sigma}=\tanh \left(\beta_{\sigma} \alpha \bar{m}_{\sigma}+\beta_{\sigma \tau}(1-\alpha) \bar{m}_{\tau}\right), \\
\bar{m}_{\tau}=\tanh \left(\beta_{\sigma \tau} \alpha \bar{m}_{\sigma}+\beta_{\tau}(1-\alpha) \bar{m}_{\tau}\right) .
\end{array}\right.
$$

Remark 1. Equations (3) can be obtained by extremizing the free energy expressed in Theorem 1 with respect to the trial parameters $\bar{m}_{\sigma}$ and $\bar{m}_{\tau}$. We stress that where $\beta_{\sigma} \beta_{\tau} \geq \beta_{\sigma \tau}^{2}$ the optimal parameters impose a maximum for the pressure landscape while in the opposite region it is a saddle point only. On the critical surface $\beta_{\sigma} \beta_{\tau}=\beta_{\sigma \tau}^{2}$ the pressure has a flat direction and the model can be described through a single order parameter that is a linear combination of the two magnetizations, i.e. $\bar{\epsilon}=\sqrt{\beta_{\sigma}} \alpha \bar{m}_{\sigma}+\sqrt{\beta_{\tau}}(1-\alpha) \bar{m}_{\tau}$ and

$$
A(\boldsymbol{\beta}, \alpha)=\ln 2+\alpha \ln \cosh \left(\sqrt{\beta_{\sigma}} \bar{\epsilon}\right)+(1-\alpha) \ln \cosh \left(\sqrt{\beta_{\tau}} \bar{\epsilon}\right)-\frac{\bar{\epsilon}^{2}}{2}
$$

with $\bar{\epsilon}$ satisfying

$$
\bar{\epsilon}=\sqrt{\beta_{\sigma}} \alpha \tanh \left(\sqrt{\beta_{\sigma}} \bar{\epsilon}\right)+\sqrt{\beta_{\tau}}(1-\alpha) \tanh \left(\sqrt{\beta_{\tau}} \bar{\epsilon}\right) .
$$

It is worth noticing that in the limit of $\beta_{\sigma \tau}=0$ the two parties are non interacting and a convex linear combination of two standard Curie-Weiss pressure at suitable temperatures is obtained, while, for $\beta_{\sigma}=\beta_{\tau}=0$, the results developed in [6] for a bipartite system without monopartite interactions are recovered.

\subsection{First approach: Sum rule}

The method that in this section we adapt to fully interacting bipartite ferromagnets has been successfully applied in [27, 10] for a huge class of single disordered system or systems in reciprocal interactions but without self-contributions. Here we show how it works in the larger case of complete topological interactions, starting with simpler case of the ferromagnetic couplings highlighting the perspective we want to follow. In the second half of the manuscript we will apply it to the disordered counterpart, which will require some more mathematical efforts. In a quick introductional summary, the technique consists of three steps:

- Through the introduction of an interpolating parameter $t \in[0,1]$, a new trial Hamiltonian is defined as the sum of two pieces, to which it reduces in the limit $t \rightarrow 1$ and $t \rightarrow 0$ : the former is the original model, which has to be solved, and the latter is spin system with a simpler one-body interaction with an external effective field that mirrors the real microscopic interactions in a pure mean field fashion, hence

$$
H(t)=(t) H_{\text {original }}+(1-t) H_{\text {one-body }} .
$$

From the interpolating Hamiltonian, the definitions of interpolating partition function $Z_{N}(t)$ and pressure $A_{N}(t)$ naturally follow simply shifting $\exp (-\beta H) \rightarrow \exp (-\beta H(t))$.

- Once the interpolating structure is defined, an interpolating procedure is needed: this role is played by the Fundamental Theorem of Calculus. The key point is that the pressure of the original model can be written as

$$
A_{N}=A_{N}(1)=A_{N}(0)+\int_{0}^{1} \frac{\partial A_{N}}{\partial t} d t .
$$

In this way the problem is split into the calculation of two terms: $A_{N}(0)$ and $\int_{0}^{1} \frac{\partial A_{N}(t)}{\partial t} d t$ 

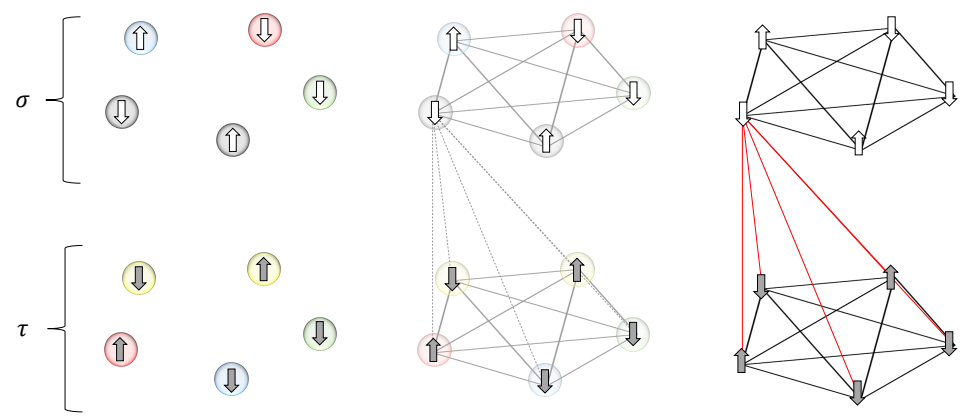

Figure 1: Schematic representation of the morphism we perform through interpolation in the sum rule (first technique). The real system is the one on the right, which is obtained whenever $t=1$ is set, while on the left the system at $t=0$ is shown. Note that at $t=0$ sites are no longer communicating, and their reciprocal interactions are replaced by effective local fields, which are represented as colored surrounding spheres (different colors represent different fields). In the middle an intermediate situation with a generic $t$ is shown for the sake of completeness.

- $A_{N}(0)$ can be easily calculated because of the factorizability property of a one-body interaction. For what concerns $\frac{\partial A_{N}(t)}{\partial t}$, it can be written as the sum of a term $\bar{A}$ independent by $t$ and a rest $R(t)$ proportional to the fluctuations of an appropriately chosen order parameter, in such a way that

$$
A_{N}=\left(A_{N}(0)+\bar{A}\right)+\int_{0}^{1} R(t) d t
$$

where $R(t)$ is the rest including all the fluctuations which one would like to delete or to reduce as much as possible, using the self-averaging property of the order parameters, when it occurs.

In the concrete case of the ferromagnetic model introduced in the previous section (eq.(1)), we define the interpolating Hamiltonian as

$$
H_{N}(t)=-t\left[\frac{1}{N} \beta_{\sigma \tau} \sum_{i=1}^{N_{\sigma}} \sum_{j=1}^{N_{\tau}} \sigma_{i} \tau_{j}+\frac{1}{2 N} \beta_{\sigma} \sum_{i, j}^{N_{\sigma}} \sigma_{i} \sigma_{j}+\frac{1}{2 N} \beta_{\tau} \sum_{i, j}^{N_{\tau}} \tau_{i} \tau_{j}\right]-(1-t)\left[C_{\sigma} \sum_{i=1}^{N_{\sigma}} \sigma_{i}+C_{\tau} \sum_{i=1}^{N_{\tau}} \tau_{i}\right],
$$

where $C_{\sigma}$ and $C_{\tau}$ are constants that have to be determined a posteriori. At $t=0$ the intensive pressure can be easily be computed as

$$
\begin{aligned}
A_{N}(0) & =\frac{1}{N} \ln Z_{N}(0)=\frac{1}{N} \ln \left[\sum_{\sigma, \tau} e^{-H_{N}(0)}\right] \\
& =\frac{1}{N} \ln \left[\left(\prod_{i=1}^{N_{\sigma}} \sum_{\sigma_{i}} e^{C_{\sigma} \sigma_{i}}\right)\left(\prod_{i=1}^{N_{\tau}} \sum_{\tau_{i}} e^{C_{\tau} \tau_{i}}\right)\right]=\ln 2+\alpha \ln \cosh \left(C_{\sigma}\right)+(1-\alpha) \ln \cosh \left(C_{\tau}\right)
\end{aligned}
$$

Then, the derivative of the pressure with respect to the interpolating parameter is performed as

$$
\begin{aligned}
\frac{\partial A_{N}(t)}{\partial t} & =\frac{1}{N} \frac{\partial}{\partial t}\left[\ln Z_{N}(t)\right]=\frac{1}{N} \frac{1}{Z_{N}(t)} \sum_{\sigma, \tau} \frac{\partial}{\partial t} e^{-H_{N}(t)} \\
& =\beta_{\sigma \tau} \alpha(1-\alpha)\left\langle m_{\sigma} m_{\tau}\right\rangle_{t}+\frac{\beta_{\sigma} \alpha^{2}}{2}\left\langle m_{\sigma}^{2}\right\rangle_{t}+\frac{\beta_{\tau}(1-\alpha)^{2}}{2}\left\langle m_{\tau}^{2}\right\rangle_{t}-C_{\sigma} \alpha\left\langle m_{\sigma}\right\rangle_{t}-C_{\tau}(1-\alpha)\left\langle m_{\tau}\right\rangle_{t}(8)
\end{aligned}
$$


Now the last expression has to be written in terms of the fluctuations of the order parameters. Defining $a, b$ and $c$ as free coefficients, the generic form of the fluctuations of the order parameters is

$$
\begin{aligned}
& a\left\langle\left(m_{\sigma}-\bar{m}_{\sigma}\right)\left(m_{\tau}-\bar{m}_{\tau}\right)\right\rangle_{t}+b\left\langle\left(m_{\sigma}-\bar{m}_{\sigma}\right)^{2}\right\rangle_{t}+c\left\langle\left(m_{\tau}-\bar{m}_{\tau}\right)^{2}\right\rangle_{t}= \\
& =a\left\langle m_{\sigma} m_{\tau}\right\rangle_{t}+b\left\langle m_{\sigma}^{2}\right\rangle_{t}+c\left\langle m_{\tau}^{2}\right\rangle_{t}+\left(-a \bar{m}_{\tau}-2 b \bar{m}_{\sigma}\right)\left\langle m_{\sigma}\right\rangle_{t}+\left(-a \bar{m}_{\sigma}-2 c \bar{m}_{\tau}\right)\left\langle m_{\tau}\right\rangle_{t}+\left[a \bar{m}_{\sigma} \bar{m}_{\tau}+b \bar{m}_{\sigma}^{2}+c \bar{m}_{\tau}^{2}\right] .
\end{aligned}
$$

Hence, we can identify each coefficient of the equation $(9)$ with the ones of the specific expression (8), in such a way that we can fix the coefficients $C_{\sigma}$ and $C_{\tau}$ as

$$
C_{\sigma}=\alpha \beta_{\sigma} \bar{m}_{\sigma}+\beta_{\sigma \tau}(1-\alpha) \bar{m}_{\tau} \quad ; \quad C_{\tau}=\beta_{\sigma \tau} \alpha \bar{m}_{\sigma}+(1-\alpha) \beta_{\tau} \bar{m}_{\tau}
$$

Using equations (7) and (8) we can then write down the following sum rule

$$
\begin{aligned}
A_{N}(\boldsymbol{\beta}, \alpha) & =\ln 2+\alpha \ln \cosh \left(\beta_{\sigma} \alpha \bar{m}_{\sigma}+\beta_{\sigma \tau}(1-\alpha) \bar{m}_{\tau}\right)+(1-\alpha) \ln \cosh \left(\beta_{\sigma \tau} \alpha \bar{m}_{\sigma}+\beta_{\tau}(1-\alpha) \bar{m}_{\tau}\right) \\
& -\left[\beta_{\sigma \tau} \alpha(1-\alpha) \bar{m}_{\sigma} \bar{m}_{\tau}+\frac{1}{2} \beta_{\sigma} \alpha^{2} \bar{m}_{\sigma}^{2}+\frac{1}{2} \beta_{\tau}(1-\alpha)^{2} \bar{m}_{\tau}^{2}\right]+R_{N}(t)
\end{aligned}
$$

where

$$
R_{N}(t)=\int_{0}^{1} d t\left[a\left\langle\left(m_{\sigma}-\bar{m}_{\sigma}\right)\left(m_{\tau}-\bar{m}_{\tau}\right)\right\rangle_{t}+b\left\langle\left(m_{\sigma}-\bar{m}_{\sigma}\right)^{2}\right\rangle_{t}+c\left\langle\left(m_{\tau}-\bar{m}_{\tau}\right)^{2}\right\rangle_{t}\right] .
$$

Since in the ferromagnetic models the magnetizations are self-averaging in the thermodynamic limit, we can argue that, for a particular choice of the parameters $\bar{m}_{\sigma}$ and $\bar{m}_{\tau}$ (that is by extremizing the pressure with respect to them) we can neglect the rest in 10, namely

$$
\lim _{N \rightarrow \infty} R_{N}(t)=0,
$$

and in the same limit $A_{N} \rightarrow A$ (where $A$ represents the pressure evaluated for $N \rightarrow \infty$ ), that completes the proof of Theorem 1 . Note that, by deriving equation $(10)$ with respect to $\bar{m}_{\sigma}$ and $\bar{m}_{\tau}$ we get

$$
\left\{\begin{array}{l}
\frac{\partial A}{\partial \bar{m}_{\sigma}}=\beta_{\sigma} \alpha^{2}\left(\left\langle\sigma_{i}\right\rangle_{t=0}-\bar{m}_{\sigma}\right)+\beta_{\sigma \tau} \alpha(1-\alpha)\left(\left\langle\tau_{i}\right\rangle_{t=0}-\bar{m}_{\tau}\right)=0, \\
\frac{\partial A}{\partial \bar{m}_{\tau}}=\beta_{\sigma \tau} \alpha(1-\alpha)\left(\left\langle\sigma_{i}\right\rangle_{t=0}-\bar{m}_{\sigma}\right)+\beta_{\tau}(1-\alpha)^{2}\left(\left\langle\tau_{i}\right\rangle_{t=0}-\bar{m}_{\tau}\right)=0
\end{array}\right.
$$

from which we can argue that, as soon as $\beta_{\sigma} \beta_{\tau} \neq \beta_{\sigma \tau}^{2}$, the optimal order parameters satisfy

$$
\begin{aligned}
& \bar{m}_{\sigma}=\left\langle\sigma_{i}\right\rangle_{t=0} \\
& \bar{m}_{\tau}=\left\langle\tau_{i}\right\rangle_{t=0},
\end{aligned}
$$

i.e. the magnetizations of the interpolating system at $t=0$ are the same of the original system's ones. From the equations 12 we can see that, on the critical surface $\beta_{\sigma} \beta_{\tau}=\beta_{\sigma \tau}^{2}$, we have just one single degenerate self consistent equation, that is eq. 5 for an order parameter $\epsilon(\boldsymbol{\sigma}, \boldsymbol{\tau})=\sqrt{\beta_{\sigma}} \alpha m_{\sigma}(\boldsymbol{\sigma}, \boldsymbol{\tau})+\sqrt{\beta_{\tau}}(1-\alpha) m_{\tau}(\boldsymbol{\sigma}, \boldsymbol{\tau})$ which is a linear combination of the two magnetizations. In this region of the phase space $\epsilon(\boldsymbol{\sigma}, \tau)$ is self averaging but the two magnetizations can fluctuate. This phenomenon is very clear for example in the special case in which $\beta_{\sigma}=\beta_{\tau}=\beta_{\sigma \tau}=\beta$, where we cannot distinguish any longer between the two parties: the system is a single Curie Wiess model, of size $N$, characterized by a single order parameter that is the global magnetization $M(\boldsymbol{\sigma}, \boldsymbol{\tau})=\alpha m_{\sigma}(\boldsymbol{\sigma})+(1-\alpha) m_{\tau}(\boldsymbol{\tau})=\beta^{-1 / 2} \epsilon(\boldsymbol{\sigma}, \boldsymbol{\tau})$.

\subsection{Second approach: The Hamilton-Jacobi framework}

Besides the fundamental theorem of calculus, another interpolation method, developed in [28], can be used. The latter is based on a mechanistic interpretation of the statistical mechanic and thermodynamic quantities defined at the beginning of this section.

The main idea is the following: the problem of obtaining an explicit expression for the pressure of the model 
(1) in the thermodynamic limit and in terms of its order and tunable parameters, is translated in solving an Hamilton-Jacobi equation, where the pressure plays as the action, with suitable boundary conditions. For this purpose, with the freedom of thinking at the interpolating parameters $t \in \mathcal{R}^{+}$and $x \in \mathcal{R}$ as fictitious time and space respectively, we first define an interpolating Hamiltonian as

$$
\begin{aligned}
H_{N}(t, x)= & -t\left[N_{\tau} \alpha \beta_{\sigma \tau} m_{\sigma} m_{\tau}+\frac{N_{\sigma}}{2} \alpha \beta_{\sigma} m_{\sigma}^{2}+\frac{N_{\tau}}{2}(1-\alpha) \beta_{\tau} m_{\tau}^{2}\right] \\
& -(1-t)\left[\frac{N_{\sigma}}{2} \beta_{\sigma}^{\prime} m_{\sigma}^{2}+\frac{N_{\tau}}{2} \beta_{\tau}^{\prime} m_{\tau}^{2}\right]-x N D_{N}(\sigma, \tau),
\end{aligned}
$$

where, introducing a free parameter $a \in(0, \infty)$ (whose practical convenience will be evident later), we defined

$$
\beta_{\sigma}^{\prime}=\alpha\left[a^{2} \beta_{\sigma \tau}+\beta_{\sigma}\right] \quad ; \quad \beta_{\tau}^{\prime}=(1-\alpha)\left[a^{-2} \beta_{\sigma \tau}+\beta_{\tau}\right],
$$

and the order parameter $D(\sigma, \tau)$ (that is just a linear combination of the magnetizations)

$$
D(\sigma, \tau)=\sqrt{\beta_{\sigma \tau}}\left[\alpha a m_{\sigma}-(1-\alpha) a^{-1} m_{\tau}\right] .
$$

Then, from the definition of the interpolating Hamiltonian we introduce, as usual, an interpolating pressure $S_{N}(t, x)=N^{-1} \ln \sum_{\sigma \tau} e^{-H_{N}(t, x)}$, which we named $S$ as it plays the role of an action in the $(t, x)$ space.

Performing the temporal and spatial derivatives of $S_{N}(t, x)$ and denoting with a subscript $(t, x)$ the averages performed within the extended Boltzmann measure weighted by $H_{N}(t, x)$, we get

$$
\begin{aligned}
\frac{\partial S_{N}(t, x)}{\partial t} & =-\frac{1}{2}\left\langle D^{2}\right\rangle_{(t, x)} \\
\frac{\partial S_{N}(t, x)}{\partial x} & =\langle D\rangle_{(t, x)} \\
\frac{\partial^{2} S_{N}(t, x)}{\partial x^{2}} & =N\left(\left\langle D^{2}\right\rangle_{(t, x)}-\langle D\rangle_{(t, x)}^{2}\right),
\end{aligned}
$$

thus, directly by construction, we can write the following Hamilton-Jacobi equation for $S_{N}(t, x)$ :

$$
\partial_{t} S_{N}(t, x)+\frac{1}{2}\left(\partial_{x} S_{N}(t, x)\right)^{2}+V_{N}(t, x)=0,
$$

where the potential $V_{N}(t, x)$ is defined as

$$
V_{N}(t, x)=-\frac{1}{2}\left(\left\langle D^{2}\right\rangle_{(t, x)}-\langle D\rangle_{(t, x)}^{2}\right)=\frac{1}{2 N} \frac{\partial^{2} S_{N}(t, x)}{\partial x^{2}} .
$$

Because of the self-averaging of the order parameter $2^{2}$ the potential becomes negligible when the size of the system grows to infinity, hence $S(t, x)$ satisfies, in the thermodynamic limit, a free Hamilton-Jacobi equation. We can easily solve it by noting that the velocity field $D(t, x)=\partial_{x} S(t, x)=\langle D\rangle_{(t, x)}$ is constant along the trajectories $x=x_{0}+D(t, x) t$, such that $D(t, x)$ can be determined from the relation

$$
D(t, x)=D\left(0, x_{0}\right)=\partial_{x} S(0, x) \mid x=x_{0}(t, x)
$$

that plays as a self consistent equation for $D$.

The general expression for the action $S(t, x)$ can be obtained as its value evaluated in a point $S\left(0, x_{0}\right)($ the Cauchy condition) plus the integral of the Lagrangian $\mathcal{L}(t, x)$ over time. Note that here, as the trajectories of such a fictitious motion are straight lines, or alternatively because the potential is zero, the Lagrangian reads off simply as $\mathcal{L}(t, x)=\langle D\rangle_{t, x}^{2} / 2$, hence overall we can write

$$
S(t, x)=S\left(t=0, x=x_{0}\right)+\int_{0}^{t} \mathcal{L}\left(t^{\prime}, x\right) d t^{\prime}=S\left(0, x_{0}\right)+\frac{t}{2}\left\langle D^{2}\right\rangle_{(t, x)} .
$$

\footnotetext{
${ }^{1}$ Note that this extended average reduces to the canonical one whenever measured at $t=1$ and $x=0$.

${ }^{2}$ Alternatively, instead of assuming self-averaging for the vector $\langle D\rangle$ in the thermodynamic $\operatorname{limit}\left(\langle D\rangle=\lim _{N \rightarrow \infty}\left\langle D_{N}\right\rangle\right)$, it is possible to obtain it simply by noticing the $N^{-1}$ pre-factor at the r.h.s. of equation 17 , multiplying a bounded function.
} 
Note also that the Cauchy starting point implicitly allows for factorization over the sites $\sigma, \tau$ as at $t=0$ the (potentially tricky) two-body interactions disappear.

All the quantities we need can then be derived simply by computing the interpolating pressure at $t=0$, which is

$$
S_{N}(0, x)=\frac{1}{N} \ln \sum_{\sigma, \tau} e^{\left(\beta_{\sigma}^{\prime} \frac{N_{\sigma}}{2} m_{\sigma}^{2}+\beta_{\tau}^{\prime} \frac{N_{\tau}}{2} m_{\tau}^{2}\right)} e^{\left(x \sqrt{\beta_{\sigma \tau}}\right) N\left(\alpha a m_{\sigma}-\left((1-\alpha) a^{-1}\right) m_{\tau}\right)}
$$

that is the pressure of two independent Curie-Wiess model with external fields $h$, i.e.

$$
S(0, x)=\alpha A^{C W}\left(\beta_{\sigma}^{\prime}, x a \sqrt{\beta_{\sigma \tau}}\right)+(1-\alpha) A^{C W}\left(\beta_{\tau}^{\prime},-x a^{-1} \sqrt{\beta_{\sigma \tau}}\right),
$$

where

$$
A^{C W}(\beta, h)=\log 2+\log \cosh (\beta(m+h))-\frac{\beta}{2} m^{2}
$$

and $m=m(\beta)$ is the solution of the self-consistent equation $m=\tanh (\beta m)$. Taking the derivative with respect to $x$ we get the initial condition for the velocity field

$$
D(0, x)=\partial_{x} S(0, x)=\sqrt{\beta_{\sigma \tau}}\left[\alpha a m\left(\beta_{\sigma}^{\prime}, x a \sqrt{\beta_{\sigma, \tau}}\right)-(1-\alpha) a^{-1} m\left(\beta_{\tau}^{\prime},-x a^{-1} \sqrt{\beta_{\sigma \tau}}\right)\right] .
$$

At this point we can explicitely write down the self-consistent equation for the velocity field $D(t, x)$ that has to be the solution of

$$
D(t, x)=D\left(0, x_{0}\right)=\sqrt{\beta_{\sigma \tau}}\left[\alpha a m\left(\beta_{\sigma}^{\prime},(x-D(t, x) t) a \sqrt{\beta_{\sigma, \tau}}\right)-(1-\alpha) a^{-1} m\left(\beta_{\tau}^{\prime},-(x-D(t, x) t) a^{-1} \sqrt{\beta_{\sigma \tau}}\right)\right] .
$$

Finally, remembering that $x_{0}=x-D(t, x) t$, the pressure of the model can be written in terms of $D(t, x)$ as

$$
S(t, x)=S(0, x-D(x, t) t)+\frac{t}{2} D^{2}(t, x) .
$$

It is easy to check that, whenever evaluated at $t=1$ and $x=0$ the expression 25 does coincide with the expression (2) obtained through the first method. In fact, referring to equation (24), we can define

$$
\begin{aligned}
& \bar{m}_{\sigma}(\boldsymbol{\beta} ; a)=m\left(\beta_{\sigma}^{\prime},-D(1,0) a \sqrt{\beta_{\sigma, \tau}}\right) \\
& \bar{m}_{\tau}(\boldsymbol{\beta} ; a)=m\left(\beta_{\tau}^{\prime}, D(1,0) a^{-1} \sqrt{\beta_{\sigma \tau}}\right) .
\end{aligned}
$$

Since $D(\boldsymbol{\beta})=D(1,0)=\sqrt{\beta_{\sigma \tau}}\left[\alpha a \bar{m}_{\sigma}-\left(1-\alpha a^{-1} m_{\tau}\right)\right], \bar{m}_{\sigma}$ and $\bar{m}_{\tau}$ satisfy the following system of coupled equations

$$
\begin{aligned}
& \bar{m}_{\sigma}=m\left(\beta_{\sigma}^{\prime},-D a \sqrt{\beta_{\sigma, \tau}}\right)=\tanh \left(\alpha \beta_{\sigma} \bar{m}_{\sigma}+(1-\alpha) \beta_{\sigma \tau} \bar{m}_{\tau}\right), \\
& \bar{m}_{\tau}=m\left(\beta_{\tau}^{\prime}, D a^{-1} \sqrt{\beta_{\sigma, \tau}}\right)=\tanh \left((1-\alpha) \beta_{\tau} \bar{m}_{\tau}+\alpha \beta_{\sigma \tau} \bar{m}_{\sigma}\right),
\end{aligned}
$$

that is exactly the system defining the order parameters in the first method and reported in eq. (3). Using this decomposition for $D(\boldsymbol{\beta})$ we can rewrite equation(25) once again obtaining for the pressure the expression (2), in full agreement with Theorem 1 statements.

Note that, as it should be, since $\bar{m}_{\sigma}(\boldsymbol{\beta} ; a)$ and $\bar{m}_{\tau}(\boldsymbol{\beta} ; a)$ do not depend on $a$, we get the same expression for the pressure of the model independently by the choice of $a$ in the interpolating procedure. This degree of freedom allows us to give a physical interpretation to the quantities $\bar{m}_{\sigma}$ and $\bar{m}_{\tau}$ as magnetizations "completely inside" the Hamilton-Jacobi framework. In fact, since

$$
D(\boldsymbol{\beta})=\sqrt{\beta_{\sigma \tau}}\left[\alpha a\left\langle m_{\sigma}\right\rangle-(1-\alpha) a^{-1}\left\langle m_{\tau}\right\rangle\right]=\sqrt{\beta_{\sigma \tau}}\left[\alpha a \bar{m}_{\sigma}-(1-\alpha) a^{-1} \bar{m}_{\tau}\right],
$$

for every choice of the parameter $a$, we obtain $\left\langle m_{\sigma}\right\rangle=\bar{m}_{\sigma}$ and $\left\langle m_{\tau}\right\rangle=\bar{m}_{\tau}$. 
Remark 2. We can use fruitfully the freedom in the choice of the free parameter a by imposing that the velocity is zero when $x=0$. In this way $S(t, x)=S\left(0, x_{0}\right)$, i.e. the pressure of the model can be written as a convex linear combination of two non interacting single-party systems at suitable temperatures. We can do that by imposing

$$
\sqrt{\beta_{\sigma \tau}}\left[\alpha a \bar{m}_{\sigma}-(1-\alpha) a^{-1} \bar{m}_{\tau}\right]=0
$$

i.e. choosing $a=\sqrt{\frac{(1-\alpha) \bar{m}_{\tau}}{\alpha \bar{m}_{\sigma}}}$. In this way we can write

$$
A\left(\beta_{\sigma}, \beta_{\tau}, \beta_{\sigma \tau}\right)=\alpha A^{C W}\left(\beta_{\sigma}^{\prime}\right)+(1-\alpha) A^{C W}\left(\beta_{\tau}^{\prime}\right)
$$

This result generalizes the decomposition introduced for the first time in [6], concerning the bipartite systems without self interactions.

\subsection{Third approach: The Fourier framework}

In line with the precedent remark, in this section we show a strategy easily obtainable revisiting the HamiltonJacobi scheme. In fact, instead of giving the solution of the model through $(24)$ and $(25)$, the (Cole-Hopf transform of the) function $S_{N}(t, x)$ can be studied in its conjugate Fourier space $(t, k)$ and solved via standard Green function plus convolution theorem route as summarized in the following adaptation of the Lax Theorem 34 .

Theorem 2. Using $S_{0}(x)$ to quantify the value of the action at $t=0$, and a subscript $N$ to denote averages of observable performed at finite $N$ with its lacking accounting for quantities evaluated in the thermodynamic limit, then for $N \rightarrow \infty$ the solution of

$$
\left\{\begin{array}{l}
\partial_{t} S_{N}(t, x)+\frac{1}{2}\left(\partial_{x} S_{N}(t, x)\right)^{2}+\frac{1}{2 N} \partial_{x}^{2} S_{N}(t, x)=0 \\
S_{N}(0, x)=S_{0}(x)
\end{array}\right.
$$

namely an explicit expression for the action $S(t, x)$, and the associated Burger problem

$$
\left\{\begin{array}{l}
\partial_{t} D_{N}(t, x)+D_{N}(t, x) \partial_{x} D_{N}(t, x)+\frac{1}{2 N} \partial_{x}^{2} D_{N}(t, x)=0, \\
D_{N}(0, x)=D_{0}(x)
\end{array}\right.
$$

is given by the Legendre transform of its Cauchy condition on the action, hence

$$
S(t, x)=\inf _{y}\left\{\frac{(x-y)^{2}}{2 t}+S_{0}(y)\right\}=\frac{(x-\hat{y})^{2}}{2 t}+S_{0}(\hat{y}),
$$

with $\hat{y}$ minimizer and $x=\hat{y}+D_{0}(\hat{y}) t$.

Proof. First we perform the following Cole-Hopf transform on $S_{N}(t, x)$

$$
\Psi_{N}(t, x):=e^{-N S_{N}(t, x)},
$$

that, by definition, satisfies the following heat equation:

$$
\frac{\partial \Psi_{N}(t, x)}{\partial t}-\frac{1}{2 N} \frac{\partial^{2} \Psi_{N}(t, x)}{\partial x^{2}}=0
$$

Now, calling its Fourier transform $\hat{\Psi}_{N}(t, k)$, clearly

$$
\partial_{t} \hat{\Psi}_{N}(t, k)+\frac{k^{2}}{2 N} \hat{\Psi}_{N}(t, k)=0,
$$


whose solution is given by

$$
\hat{\Psi}_{N}(t, k)=\hat{\Psi}_{0}(k) \exp \left(-\frac{k^{2}}{2 N} t\right)
$$

Coming back to the original space we get

$$
\Psi_{N}(t, x)=\int d y G_{t}(x-y) \Psi_{0}(y)=\sqrt{\frac{N}{2 \pi t}} \int d y e^{-N \frac{(x-y)^{2}}{2 t}} \Psi_{0}(y)
$$

where $G_{t}(x-y)$ is the Green propagator. Recalling the definition of $\Psi_{N}(t, x)$ we get

$$
S_{N}(t, x)=-\frac{1}{N} \log \Psi_{N}(t, x)=-\frac{1}{N} \ln \sqrt{\frac{N}{2 \pi t}} \int d y e^{-N\left(\frac{(x-y)^{2}}{2 t}+S_{0}(y)\right)}
$$

that can be computed in the thermodynamic limit through the saddle-point technique obtaining

$$
S(t, x)=\inf _{y}\left\{\frac{(x-y)^{2}}{2 t}+S_{0}(y)\right\} .
$$

Using the explicit definition of $S(t, x)$, once computed the initial condition 21), we can use Lemma 2 and recover exactly equation 25 from which all the considerations of the previous section hold.

\section{Disordered case: Replica Symmetric Approximation}

\subsection{The Model}

In this second part of the paper we study a fully interacting bipartite spin glass. Namely we investigate the disordered counterpart of the model (1) where now the coupling may assume both positive and negative values allowing for frustration. Thus, besides a different normalization of the Hamiltonian in order to ensure the standard extensive linear growth of the thermodynamical observables with the size of the system, the exchange interactions now are independently drawn at random from a Gaussian distribution $\mathcal{N}(0,1)$, hence

$$
H_{N}(\sigma, \tau ; \mathbf{J})=-\frac{1}{\sqrt{N}} \beta_{\sigma \tau} \sum_{i=1}^{N_{\sigma}} \sum_{j=1}^{N_{\tau}} J_{i j}^{\sigma \tau} \sigma_{i} \tau_{j}-\frac{1}{\sqrt{2 N_{\sigma}}} \beta_{\sigma} \sum_{i, j}^{N_{\sigma}} J_{i j}^{\sigma} \sigma_{i} \sigma_{j}-\frac{1}{\sqrt{2 N_{\tau}}} \beta_{\tau} \sum_{i, j}^{N_{\tau}} J_{i j}^{\tau} \tau_{i} \tau_{j},
$$

The factor $1 / \sqrt{2}$, when present, ensures the contribution of each couple of spins to count just once. Further, as in the ferromagnetic case, each contribution is weighted with a $\beta$-parameter, modulating the relative strength between interactions of different nature (mono-partite or bipartite) and within each party. Then, one can define easily the statistical mechanics machinery as before, this time introducing replicas too. Thus, using $\mathbb{E}$ to depict the average over the Gaussian couplings, we have:

Partition function

Boltzmann average

Product measure over $S$ replicas

Quenched state

Overlap of the $\sigma$ party

Overlap of the $\tau$ party

Quenched intensive pressure

$$
\begin{gathered}
Z_{N}=\sum_{\{\sigma, \tau\}} e^{-\beta H_{N}(\sigma, \tau ; \mathbf{J})} \\
\omega_{N}(\mathcal{O} ; \mathbf{J})=Z_{N}^{-1} \sum_{\{\sigma, \tau\}} \mathcal{O} e^{-\beta H_{N}(\sigma, \tau ; \mathbf{J})} \\
\Omega=\omega_{1} \otimes \ldots \otimes \omega_{s} \\
\langle\mathcal{O}\rangle=\mathbb{E}[\Omega(\mathcal{O})] \\
q_{\sigma^{a} \sigma^{b}}=\frac{1}{N_{\sigma}} \sum_{i} \sigma_{i}^{a} \sigma_{i}^{b} \\
q_{\tau^{a} \tau^{b}}=\frac{1}{N_{\tau}} \sum_{\mu} \tau_{i}^{a} \tau_{i}^{b}
\end{gathered}
$$


As usual the (quenched) free energy $f(\alpha, \beta)$ is related to the (quenched) pressure $A(\alpha, \beta)$ via $A(\alpha, \beta)=$ $-\beta f(\alpha, \beta)$. Note that in the rest of the paper we will set $\beta=1$ without loss of generality as we can restore the dependence by $\beta$ simply rescaling the couplings $\beta_{x} \rightarrow \beta \beta_{x}$, with $x=\sigma, \tau, \sigma \tau$. As in the first part of the paper, the expression of the quenched pressure in the replica symmetric approximation is determined using the three different techniques described before. Nevertheless, the presence of the overlaps instead of the magnetizations of the spins implies slightly different procedures in the proofs with respect to the ferromagnetic case. Even so, all the strategies produce the same solution as stated in the following

Theorem 3. The Replica Symmetric Approximation for the intensive pressure of the model defined in (38) reads as

$$
\begin{aligned}
& A^{R S}(\alpha, \boldsymbol{\beta})=\ln 2 \\
& +\alpha \int d \mu(z) \ln \cosh \left(z \sqrt{\left(\beta_{\sigma}^{2}\right) \bar{q}_{\sigma \sigma^{\prime}}+\beta_{\sigma \tau}^{2}(1-\alpha) \bar{q}_{\tau \tau^{\prime}}}\right)+(1-\alpha) \int d \mu(z) \ln \cosh \left(z \sqrt{\left(\beta_{\sigma \tau}^{2} \alpha\right) \bar{q}_{\sigma \sigma^{\prime}}+\left(\beta_{\tau}^{2}\right) \bar{q}_{\tau \tau^{\prime}}}\right)+ \\
& +\frac{\beta_{\sigma}^{2}}{4} \alpha\left(\bar{q}_{\sigma \sigma^{\prime}}-1\right)^{2}+\frac{\beta_{\tau}^{2}}{4}(1-\alpha)\left(\bar{q}_{\tau \tau^{\prime}}-1\right)^{2}+\frac{1}{2} \beta_{\sigma \tau}^{2} \alpha(1-\alpha)\left(1-\bar{q}_{\sigma \sigma^{\prime}}\right)\left(1-\bar{q}_{\tau \tau^{\prime}}\right),
\end{aligned}
$$

where $d \mu(z)$ is a unitary gaussian measure and the order parameters $\bar{q}_{\sigma \sigma^{\prime}}$ and $\bar{q}_{\tau \tau^{\prime}}$ are the solutions of the following system of self-consistent coupled equations

$$
\left\{\begin{array}{l}
\bar{q}_{\sigma \sigma^{\prime}}=\int d \mu(z) \tanh ^{2}\left(z \sqrt{\beta_{\sigma}^{2} \bar{q}_{\sigma \sigma^{\prime}}+\beta_{\sigma \tau}^{2}(1-\alpha) \bar{q}_{\tau \tau^{\prime}}}\right), \\
\bar{q}_{\tau \tau^{\prime}}=\int d \mu(z) \tanh ^{2}\left(z \sqrt{\beta_{\sigma \tau}^{2} \alpha \bar{q}_{\sigma \sigma^{\prime}}+\beta_{\tau}^{2} \bar{q}_{\tau \tau^{\prime}}}\right) .
\end{array}\right.
$$

Finally, in the region

$$
\beta_{\sigma} \beta_{\tau} \geq \beta_{\sigma \tau}^{2} \sqrt{\alpha(1-\alpha)}
$$

the following sum rule holds

$$
A(\alpha, \boldsymbol{\beta}) \leq A^{R S}(\alpha, \boldsymbol{\beta})
$$

As it will be clear in the next sections, the replica symmetric approximation can be defined assuming that the fluctuations of the order parameters of the model, i.e. $\left\langle q_{\sigma \sigma^{\prime}}^{2}\right\rangle-\left\langle q_{\sigma \sigma^{\prime}}\right\rangle^{2}$ and $\left\langle q_{\tau \tau^{\prime}}^{2}\right\rangle-\left\langle q_{\tau \tau^{\prime}}\right\rangle^{2}$, can be neglected in the thermodynamic limit (hence the order parameters are self-averaging quantities). This assumption was exact in the ferromagnetic model but of course it is no longer true in the low noise region of the phase diagram for the disordered counterpart [36]: Indeed the well known phenomenon of replica symmetry breaking, clearly understood for single species [39, 40] 29, 42, occurs also in multi-specie spin-glasses [8], but a Parisi-like theory in this case is still missing, hence, we will focus only on replica symmetric regimes, which, for practical purposes, are generally the standard level of description [3, 17].

As in the ferromagnetic case, when $\beta_{\sigma \tau}=0$ the sum of two independent spin-glass replica symmetric solutions (namely of the Sherrington-Kirkpatrick (SK) type [36]) is obtained and for $\beta_{\sigma}=\beta_{\tau}=0$ the same representation of the free energy as the one shown in [6] for a purely bipartite interaction is founded.

As a last remark before proving Theorem 3 note that the condition (40], as shown in [8], plays a very important role for a lot of issues including the proof of the existence of the thermodynamic limit and the convexity of the variational principle regulating the free energy. Finally, exactly as happened in the previous ferromagnetic counterpart, we will see that, on the critical surface $\beta_{\sigma} \beta_{\tau}=\beta_{\sigma \tau}^{2} \sqrt{\alpha(1-\alpha)}$, a complete description of the model needs just one single order parameter that is a linear combination of the two overlaps: This phenomenon can be easily understood when $\beta_{\sigma} / \sqrt{\alpha}=\beta_{\tau} / \sqrt{1-\alpha}=\beta_{\sigma \tau}$, i.e. when all the interactions have the same strength: we can't distinguish the two parties and the system reduces just to a single SK spin glass model that can be described completely through a single order parameter that is the global overlap.

\subsection{First approach: Sum rule}

In this section we give a first proof of Theorem (3) by interpolating between the original model with nasty two-body couplings and a system regulated by a suitable one-body Hamiltonian whose spins feel an effective 
random external field representing -at least on average- their microscopic surrounding. This procedure allows to obtain, via the Fundamental Theorem of Calculus, a sum rule for the free energy where overlap fluctuations are embedded in a source term, split from the rest (which, as a consequence, naturally returns the replica symmetric approximation once neglected).

First of all we define the interpolating Hamiltonian as

$$
\begin{aligned}
H_{N}(t)= & -\sqrt{t}\left[\frac{\beta_{\sigma \tau}}{\sqrt{N}} \sum_{i=1}^{N_{\sigma}} \sum_{j=1}^{N_{\tau}} J_{i j}^{\sigma \tau} \sigma_{i} \tau_{j}+\frac{\beta_{\sigma}}{\sqrt{2 N_{\sigma}}} \sum_{i, j}^{N_{\sigma}} J_{i j}^{\sigma} \sigma_{i} \sigma_{j}+\frac{\beta_{\tau}}{\sqrt{2 N_{\tau}}} \sum_{i, j}^{N_{\tau}} J_{i j}^{\tau} \tau_{i} \tau_{j}\right]+ \\
& -\sqrt{1-t}\left[\sqrt{C_{\sigma}} \sum_{i} \eta_{i}^{\sigma} \sigma_{i}+\sqrt{C_{\tau}} \sum_{\tau} \eta_{i}^{\tau} \tau_{i}\right]
\end{aligned}
$$

where the $\left\{\eta_{i}^{\sigma}\right\}_{i=1, \ldots, N_{\sigma}}$ and the $\left\{\eta_{i}^{\tau}\right\}_{i=1, \ldots, N_{\tau}}$ are two families of independent unitary gaussian random variables, independent also from the $\mathbf{J}$ and $C_{\sigma}$ and $C_{\tau}$ are two constants that we have to fix appropriately. Defining naturally the interpolating partition function $Z_{N}(t)$ and the quenched pressure $A_{N}(t)$ as

$$
Z_{N}(t)=\sum_{\{\sigma\}} \sum_{\{\tau\}} e^{-H_{N}(t)} \quad A_{N}(t)=\frac{1}{N} \mathbb{E} \ln Z_{N}(t)
$$

we recover the original pressure at $t=1$ while, at $t=0$ we have a simpler one body problem that factorizes over the sites and whose pressure can be easily computed and reads as

$$
\begin{aligned}
A_{N}(0) & =\frac{1}{N} \mathbb{E} \ln Z(0)=\frac{1}{N} \mathbb{E} \ln \sum_{\{\sigma\}} \sum_{\{\tau\}} e^{-H(0)}= \\
& =\ln 2+\alpha \int d \mu(z) \ln \cosh \left(z \sqrt{C_{\sigma}}\right)+(1-\alpha) \int d \mu(z) \ln \cosh \left(z \sqrt{C_{\tau}}\right) .
\end{aligned}
$$

The calculation leading to an explicit expression of $\partial_{t} A_{N}(t)$ is long but straightforward and returns

$$
\begin{aligned}
\frac{\partial A_{N}(t)}{\partial t}= & -\left[\frac{1}{2} \beta_{\sigma \tau}^{2} \alpha(1-\alpha)\left\langle q_{\sigma \sigma^{\prime}} q_{\tau \tau^{\prime}}\right\rangle+\frac{\beta_{\sigma}^{2}}{4} \alpha\left\langle q_{\sigma \sigma^{\prime}}^{2}\right\rangle+\frac{\beta_{\tau}^{2}}{4}(1-\alpha)\left\langle q_{\tau \tau^{\prime}}^{2}\right\rangle\right] \\
& +\left[\frac{C_{\sigma}}{2} \alpha\left\langle q_{\sigma \sigma^{\prime}}\right\rangle+\frac{C_{\tau}}{2}(1-\alpha)\left\langle q_{\tau \tau^{\prime}}\right\rangle\right] \\
& +\left[-\frac{C_{\sigma}}{2} \alpha-\frac{C_{\tau}}{2}(1-\alpha)+\frac{\beta_{\tau}^{2}}{4}(1-\alpha)+\frac{\beta_{\sigma}^{2}}{4} \alpha+\frac{1}{2} \beta_{\sigma \tau}^{2} \alpha(1-\alpha)\right]
\end{aligned}
$$

Hence, if we choose

$$
C_{\sigma}=\left(\beta_{\sigma}^{2}\right) \bar{q}_{\sigma \sigma^{\prime}}+\beta_{\sigma \tau}^{2}(1-\alpha) \bar{q}_{\tau \tau^{\prime}} \quad ; \quad C_{\tau}=\left(\beta_{\sigma \tau}^{2} \alpha\right) \bar{q}_{\sigma \sigma^{\prime}}+\left(\beta_{\tau}^{2}\right) \bar{q}_{\tau \tau^{\prime}}
$$

we can write down the $t$-streaming as

$$
\begin{aligned}
\frac{\partial A_{N}(t)}{\partial t} & =\alpha \frac{\beta_{\sigma}^{2}}{4}\left(1-\bar{q}_{\sigma \sigma^{\prime}}\right)^{2}+(1-\alpha) \frac{\beta_{\tau}^{2}}{4}\left(1-\bar{q}_{\tau \tau^{\prime}}\right)^{2}+\alpha(1-\alpha) \frac{\beta_{\sigma \tau}^{2}}{2}\left(1-\bar{q}_{\sigma \sigma^{\prime}}\right)\left(1-\bar{q}_{\tau \tau^{\prime}}\right) \\
& -\left[\alpha \frac{\beta_{\sigma}^{2}}{4}\left\langle\left(q_{\sigma \sigma^{\prime}}-\bar{q}_{\sigma \sigma^{\prime}}\right)^{2}\right\rangle_{t}+(1-\alpha) \frac{\beta_{\tau}^{2}}{4}\left\langle\left(q_{\tau \tau^{\prime}}-\bar{q}_{\tau \tau^{\prime}}\right)^{2}\right\rangle_{t}+\alpha(1-\alpha) \frac{\beta_{\sigma \tau}^{2}}{2}\left\langle\left(q_{\sigma \sigma^{\prime}}-\bar{q}_{\sigma \sigma^{\prime}}\right)\left(q_{\tau \tau^{\prime}}-\bar{q}_{\tau \tau^{\prime}}\right)\right\rangle_{t}\right] .
\end{aligned}
$$

Using equation 45 and the last expression for the $t$-streaming of $A(t)$, we can then build the following sum-rule

$$
A_{N}(\alpha, \boldsymbol{\beta})=A_{N}(1)=A_{N}(0)+\int_{0}^{1} d t \frac{d}{d t} A_{N}(t)=A^{R S}\left(\bar{q}_{\sigma \sigma^{\prime}}, \bar{q}_{\tau \tau^{\prime}}\right)-\int_{0}^{1} R_{N}(t)
$$


where $A^{R S}\left(\bar{q}_{\sigma \sigma^{\prime}}, \bar{q}_{\tau \tau^{\prime}}\right)$ is the function stated in Theorem 3 for a generic couple of parameters $\bar{q}_{\sigma \sigma^{\prime}}$ and $\bar{q}_{\tau \tau^{\prime}}$, while the source of overlap fluctuations reads as the rest

$$
R_{N}(t)=\alpha \frac{\beta_{\sigma}^{2}}{4}\left\langle\left(q_{\sigma \sigma^{\prime}}-\bar{q}_{\sigma \sigma^{\prime}}\right)^{2}\right\rangle_{t}+(1-\alpha) \frac{\beta_{\tau}^{2}}{4}\left\langle\left(q_{\tau \tau^{\prime}}-\bar{q}_{\tau \tau^{\prime}}\right)^{2}\right\rangle_{t}+\alpha(1-\alpha) \frac{\beta_{\sigma \tau}^{2}}{2}\left\langle\left(q_{\sigma \sigma^{\prime}}-\bar{q}_{\sigma \sigma^{\prime}}\right)\left(q_{\tau \tau^{\prime}}-\bar{q}_{\tau \tau^{\prime}}\right)\right\rangle_{t}
$$

As soon as $\beta_{\sigma} \beta_{\tau} \geq \beta_{\sigma \tau}^{2} \sqrt{\alpha(1-\alpha)}$, such a source is positively defined and we can minimize the error we commit keeping only the replica-symmetric approximation simply by finding the values of the order parameters that minimize $A^{R S}\left(\bar{q}_{\sigma \sigma^{\prime}}, \bar{q}_{\tau \tau^{\prime}}\right)$. By extremizing $A^{R S}\left(\bar{q}_{\sigma \sigma^{\prime}}, \bar{q}_{\tau \tau^{\prime}}\right)$ with respect to $\bar{q}_{\sigma \sigma^{\prime}}$ and $\bar{q}_{\sigma \sigma^{\prime}}$ we find the conditions (39) that complete the proof of Theorem 3. Note that, in the language of the current interpolating method, the equations 39 for the order parameters can be written in the following form

$$
\begin{aligned}
\bar{q}_{\sigma \sigma^{\prime}} & =\left\langle q_{\sigma \sigma^{\prime}}\right\rangle_{t=0} \\
\bar{q}_{\tau \tau^{\prime}} & =\left\langle q_{\tau \tau^{\prime}}\right\rangle_{t=0}
\end{aligned}
$$

This means that the optimal order parameters represent the mean of the system's overlap when $t=0$. This shows a sort of stochastic stability [19] in the interpolating procedure and justifies the definition of $A^{R S}(\alpha, \boldsymbol{\beta})$ also in the region $\beta_{\sigma} \beta_{\tau} \leq \beta_{\sigma \tau}^{2} \sqrt{\alpha(1-\alpha)}$, where we don't know the sign of the error term. Finally we want just to point out that, in this interpolating framework, the name "replica symmetric approximation" is justified by the sum rule 48 , but $A^{R S}(\alpha, \boldsymbol{\beta})$ is the true pressure of the model only if the error term vanishes in the thermodynamic limit, i.e. only in the region of the phase space where the overlaps are self-averaging (high temperature limit [36]).

\subsection{Second approach: The Hamilton-Jacobi framework}

In this section, as in the ferromagnetic case, we give a proof of Theorem 3 using a mechanical analogy with an Hamilton-Jacobi problem for a free particle 3 . First of all we define a (fictitious) time and space dependent Hamiltonian

$$
\begin{aligned}
H_{N}(t, x)= & -\sqrt{t}\left[\frac{1}{\sqrt{N}} \beta_{\sigma \tau} \sum_{i=1}^{N_{\sigma}} \sum_{j=1}^{N_{\tau}} J_{i j}^{\sigma \tau} \sigma_{i} \tau_{j}+\frac{1}{\sqrt{2 N_{\sigma}}} \beta_{\sigma} \sum_{i, j}^{N_{\sigma}} J_{i j}^{\sigma} \sigma_{i} \sigma_{j}+\frac{1}{\sqrt{2 N_{\tau}}} \beta_{\tau} \sum_{i, j}^{N_{\tau}} J_{i j}^{\tau} \tau_{i} \tau_{j}\right]+ \\
& -\sqrt{1-t}\left[\frac{\sqrt{\beta_{\sigma}^{\prime}}}{\sqrt{2 N_{\sigma}}} \sum_{i, j}^{N_{\sigma}} \hat{J}_{i j}^{\sigma} \sigma_{i} \sigma_{j}+\frac{\sqrt{\beta_{\tau}^{\prime}}}{\sqrt{2 N_{\tau}}} \sum_{i, j}^{N_{\tau}} \hat{J}_{i j}^{\tau} \tau_{i} \tau_{j}\right]+ \\
& -\left(\sqrt{x \beta_{\sigma \tau}}\right)\left[\sqrt{a} \sum_{i} J_{i}^{\sigma} \sigma_{i}+\sqrt{a^{-1}} \sum_{i} J_{i}^{\tau} \tau_{i}\right],
\end{aligned}
$$

where

$$
\beta_{\sigma}^{\prime}=\beta_{\sigma}^{2}-a^{2} \alpha \beta_{\sigma \tau}^{2} \quad ; \quad \beta_{\tau}^{\prime}=\beta_{\tau}^{2}-a^{-2}(1-\alpha) \beta_{\sigma \tau}^{2},
$$

$a$ is a positive free parameter and the $\{\mathbf{J}\}$ and $\{\hat{\mathbf{J}}\}$ are all families of unitary gaussian random variable independent from each other. Then we define naturally an interpolating pressure as

$$
A_{N}(t, x)=-\beta f_{N}(\beta)=\frac{1}{N} \mathbb{E} \ln Z_{N}(t, x)=\frac{1}{N} \mathbb{E} \ln \sum_{\sigma, \tau} e^{-H_{N}(t, x)},
$$

where $f_{N}(\beta)$ is the standard quenched free energy.

Finally we define an interpolating action $S_{N}(t, x)$ that, this time, is not directly the interpolating pressure as

\footnotetext{
${ }^{3}$ Restricting to single-specie spin glasses, the phenomenon of replica symmetry breaking within the Hamilton-Jacobi framework has been solved and has been reported in [12. For multi-species spin-glasses a quantitative description of such a phenomenon is still lacking. A first trial can be found in [8].
} 
in the first part of the paper. Here, we need to add two constants that will be determined a posteriori. In other words we define

$$
S_{N}(t, x)=2 A_{N}(t, x)+X x+T t .
$$

Deriving the action with respect to $t$ we get

$$
\begin{aligned}
\frac{\partial S_{N}(t, x)}{\partial t} & =\frac{2}{N} \mathbb{E}\left[Z_{N(t, x)}^{-1} \sum_{\sigma, \tau} \frac{\partial}{\partial t} e^{-H_{N}(t, x)}\right]+T \\
& =-\frac{1}{2}\left\langle\left[\beta_{\sigma \tau}\left(\alpha a q_{\sigma \sigma^{\prime}}+(1-\alpha) a^{-1} q_{\tau \tau^{\prime}}\right)\right]^{2}\right\rangle_{(t, x)}+\frac{1}{2} \beta_{\sigma \tau}^{2}\left(\alpha a+(1-\alpha) a^{-1}\right)+T \\
& =-\frac{1}{2}\left\langle\left[\beta_{\sigma \tau}\left(\alpha a q_{\sigma \sigma^{\prime}}+(1-\alpha) a^{-1} q_{\tau \tau^{\prime}}\right)\right]^{2}\right\rangle_{(t, x)},
\end{aligned}
$$

where we have chosen $T=-\frac{1}{2} \beta_{\sigma \tau}^{2}\left(\alpha a+(1-\alpha) a^{-1}\right)$ in order to have a square product in the last expression. For the derivative with respect to the space variable we have

$$
\begin{aligned}
\frac{\partial S_{N}(t, x)}{\partial x} & =\frac{2}{N} \mathbb{E}\left[\frac{1}{Z_{N}(t, x)} \sum_{\{\sigma\}} \sum_{\{\tau\}} \frac{\partial}{\partial x} e^{-H_{N}(t, x)}\right]+X \\
& =-\left\langle\beta_{\sigma \tau}\left(\alpha a q_{\sigma \sigma^{\prime}}+(1-\alpha) a^{-1} q_{\tau \tau^{\prime}}\right)\right\rangle_{(t, x)}+\beta_{\sigma \tau}\left(a \alpha+a^{-1}(1-\alpha)\right)+X \\
& =-\left\langle\beta_{\sigma \tau}\left(\alpha a q_{\sigma \sigma^{\prime}}+(1-\alpha) a^{-1} q_{\tau \tau^{\prime}}\right)\right\rangle_{(t, x)}
\end{aligned}
$$

with the choice of $X=-\beta_{\sigma \tau}\left(a \alpha+a^{-1}(1-\alpha)\right)$. If we call, as in the ferromagnetic case, the velocity field

$$
D_{N}(t, x)=\partial_{x} S_{N}(t, x)=-\beta_{\sigma \tau}\left\langle D_{N}(\sigma, \tau ; a)\right\rangle_{(t, x)},
$$

where we defined the observable $D_{N}(\boldsymbol{\sigma}, \boldsymbol{\tau} ; a)=\alpha a q_{\sigma \sigma^{\prime}}(\boldsymbol{\sigma})+(1-\alpha) a^{-1} q_{\tau \tau^{\prime}}(\boldsymbol{\tau})$ that is a linear combination of the two overlaps, we can easily write down an Hamilton-Jacobi equation for $S_{N}(t, x)$ as

$$
\begin{aligned}
\partial_{t} S_{N}(t, x) & +\frac{1}{2}\left(\partial_{x} S_{N}(t, x)\right)^{2}+V_{N}(t, x)=0, \\
V_{N}(t, x) & =-\frac{1}{2} \beta_{\sigma \tau}^{2}\left(\left\langle D_{N}^{2}\right\rangle_{(t, x)}-\left\langle D_{N}\right\rangle_{(t, x)}^{2}\right)=0 .
\end{aligned}
$$

In contrast with the ferromagnetic case, where the potential evidently vanished in the thermodynamic limit, in the disordered case the potential $\left\langle D_{N}^{2}\right\rangle_{(t, x)}-\left\langle D_{N}\right\rangle_{(t, x)}^{2}$, proportional to the fluctuations of the order parameters, is not in general negligible, neither in the thermodynamic limit [36 28]. Still, if we are looking for a replicasymmetric approximation of the real (full-RSB) solution, we can impose $\lim _{N \rightarrow \infty} V_{N}(t, x)=0$ and try to solve a free Hamilton-Jacobi equation for $S(t, x)$. For this purpose we need to compute first the initial condition for the action ${ }^{4}$, that is

$$
\begin{aligned}
& S_{N}(0, x)=\frac{2}{N} \mathbb{E} \ln Z_{N}(0, x)+X x= \\
& =\frac{2}{N} \mathbb{E} \ln \sum_{\boldsymbol{\sigma}} e^{\sqrt{\frac{\beta_{\sigma}^{\prime}}{2 N_{\sigma}}} \sum_{i, j}^{N_{\sigma}} \hat{J}_{i j}^{\sigma} \sigma_{i} \sigma_{j}+\sqrt{\beta_{\sigma \tau} a x} \sum_{i} J_{i}^{\sigma} \sigma_{i}}+\frac{2}{N} \mathbb{E} \ln \sum_{\tau} e^{\sqrt{\frac{\beta_{\tau}^{\prime}}{2 N_{\tau}}} \sum_{i, j}^{N_{\tau}} \hat{J}_{i j}^{\tau} \tau_{i} \tau_{j}+\sqrt{\beta_{\sigma \tau} a^{-1} x} \sum_{i} J_{i}^{\tau} \tau_{i}}+X x
\end{aligned}
$$

and contains the free energies of two SK models with external random field and different temperatures $\sqrt{\beta_{\sigma}^{\prime}}$ and $\sqrt{\beta_{\tau}^{\prime}}$, i.e.

$$
S_{N}(0, x)=2 \alpha A_{N}^{S K}\left(\sqrt{\beta_{\sigma}^{\prime}}, \sqrt{\beta_{\sigma \tau} a x}\right)+2(1-\alpha) A_{N}^{S K}\left(\sqrt{\beta_{\tau}^{\prime}}, \sqrt{\beta_{\sigma \tau} a^{-1} x}\right)+X x .
$$

\footnotetext{
${ }^{4}$ Here the strength of the method becomes clearly manifest as the calculation of the Cauchy condition $S_{N}\left(t=0, x=x_{0}\right)$ implies considering only one-body interactions (that trivially factorizes) and whose analytic expression is immediate.
} 
Since we are interested in the replica symmetric approximation of the solution, we can use it also in the initial condition, replacing $A^{S K}(\beta)$ with the well known RS approximation [36]

$$
A_{R S}^{S K}(\beta)=\log 2+\int d \mu(z) \log \cosh \left(z \sqrt{\beta^{2} q}\right)+\frac{\beta^{2}}{4}(1-q)^{2}
$$

with $q=q(\beta)$ solution of the self consistent equation

$$
q(\beta)=\int d \mu(z) \tanh ^{2}\left(z \sqrt{\beta^{2} q(\beta)}\right) .
$$

As in the ferromagnetic case, taking the derivative with respect to $x$ we get the initial condition for the velocity

$$
D(0, x)=\partial_{x} S(0, x)=-2 \beta_{\sigma \tau}\left[\alpha a \int q\left(\sqrt{\beta_{\sigma}^{\prime}}, \sqrt{\beta_{\sigma \tau} a x}\right)+(1-\alpha) a^{-1} q\left(\sqrt{\beta_{\tau}^{\prime}}, \sqrt{\beta_{\sigma \tau} a^{-1} x}\right)\right],
$$

that allows us to write the following self consistent equation for $D(t, x)$ :

$$
\begin{aligned}
D(t, x) & =D\left(0, x_{0}\right)=D(0, x-D(t, x) t)= \\
& =-2 \beta_{\sigma \tau}\left[\alpha a q\left(\sqrt{\beta_{\sigma}^{\prime}}, \sqrt{\beta_{\sigma \tau} a(x-D t)}\right)+(1-\alpha) a^{-1} q\left(\sqrt{\beta_{\tau}^{\prime}}, \sqrt{\beta_{\sigma \tau} a^{-1}(x-D t)}\right)\right]
\end{aligned}
$$

and finally the solution of the model as

$$
A(t, x)=\frac{1}{2}(S(t, x)-X x-T t)=\frac{1}{2}\left(S(0, x-D(t, x) t)+\frac{1}{2} D(t, x)^{2} t-X x-T t\right) .
$$

At $t=1$ and $x=0$, when we recover the original model, the velocity field $D(\boldsymbol{\beta})=D(1,0)$ is the solution of

$$
D(\boldsymbol{\beta})=-2 \beta_{\sigma \tau}\left[\alpha a q\left(\sqrt{\beta_{\sigma}^{\prime}}, \sqrt{-\beta_{\sigma \tau} a D}\right)+(1-\alpha) a^{-1} q\left(\sqrt{\beta_{\tau}^{\prime}}, \sqrt{-\beta_{\sigma \tau} a^{-1} D}\right)\right] .
$$

If we call

$$
\begin{aligned}
& \bar{q}_{\sigma \sigma^{\prime}}(\boldsymbol{\beta} ; a)=q\left(\sqrt{\beta_{\sigma}^{\prime}}, \sqrt{-\beta_{\sigma \tau} a D}\right), \\
& \bar{q}_{\tau \tau^{\prime}}(\boldsymbol{\beta} ; a)=q\left(\sqrt{\beta_{\tau}^{\prime}}, \sqrt{-\beta_{\sigma \tau} a D}\right),
\end{aligned}
$$

since $D(\boldsymbol{\beta})=-2 \beta_{\sigma \tau}\left[\alpha a \bar{q}_{\sigma \sigma^{\prime}}(\boldsymbol{\beta} ; a)+(1-\alpha) a^{-1} \bar{q}_{\tau \tau^{\prime}}(\boldsymbol{\beta} ; a)\right]$ and using the definition of $q(\beta)$, we can easily check that $\bar{q}_{\sigma \sigma^{\prime}}$ and $\bar{q}_{\tau \tau^{\prime}}$ satisfy the following system of coupled self-consistent equation, independent by the parameter $a$,

$$
\begin{aligned}
\bar{q}_{\sigma \sigma^{\prime}} & =\int d \mu(z) \tanh ^{2}\left(z \sqrt{\beta_{\sigma}^{2} \bar{q}_{\sigma \sigma^{\prime}}+(1-\alpha) \beta_{\sigma \tau}^{2} \bar{q}_{\tau \tau^{\prime}}}\right) \\
\bar{q}_{\tau \tau^{\prime}} & =\int d \mu(z) \tanh ^{2}\left(z \sqrt{\beta_{\tau}^{2} \bar{q}_{\tau \tau^{\prime}}+\alpha \beta_{\sigma \tau}^{2} \bar{q}_{\sigma \sigma^{\prime}}}\right)
\end{aligned}
$$

that mirrors exactly what we obtained in the previous section (equation (39)). Note that, also in this case, due to the freedom in the choice of the interpolating parameter $a$, i.e.

$$
\alpha a\left\langle q_{\sigma \sigma^{\prime}}(\sigma)\right\rangle+(1-\alpha) a^{-1}\left\langle q_{\tau \tau^{\prime}}(\tau)\right\rangle=\alpha a \bar{q}_{\sigma \sigma^{\prime}}+(1-\alpha) a^{-1} \bar{q}_{\tau \tau^{\prime}},
$$

we can associate $\bar{q}_{\sigma \sigma^{\prime}}=\left\langle q_{\sigma \sigma^{\prime}}(\sigma)\right\rangle$ and $\bar{q}_{\tau \tau^{\prime}}=\left\langle q_{\tau \tau^{\prime}}(\tau)\right\rangle$ and characterize completely the model. Using the previous decomposition for $D(\boldsymbol{\beta})$ inside the equation [61), we get the free energy of the model in terms of overlaps recovering the main expression enclosed in the statements of Theorem 3. 


\subsection{Third approach: the Fourier framework}

Once introduced the mechanical interpolating scheme, we can solve the Hamilton-Jacobi equation (55) using, as in the ferromagnetic counterpart, the Fourier method too. We can do that again in the replica symmetry approximation in which we neglect the potential, proportional to the fluctuations of the order parameters.

In this context we have to note that solving a free Hamilton Jacobi equation is equivalent to solving a Burger-like equation

$$
\partial_{t} S_{N}(t, x)+\frac{1}{2}\left(\partial_{x} S_{N}(t, x)\right)^{2}+\frac{1}{2 N} \partial_{x^{2}}^{2} S_{N}(t, x)=0,
$$

where we added an irrelevant, because vanishing in the thermodynamic limit, mollifier term proportional to the second derivative of $S_{N}(t, x)$.

Hence, also for replica-symmetric bipartite spin-glasses, in this way we can apply a Cole Hopf transform, namely introduce a function $\Psi_{N}(t, x)$ as

$$
\Psi_{N}(t, x)=\exp \left(-N S_{N}(t, x)\right)
$$

Trough the latter we can map the problem of solving for the quenched pressure in statistical mechanics in solving a heat equation for the Cole-Hopf transform of the action, namely

$$
\frac{\partial \Psi_{N}(t, x)}{\partial t}-\frac{1}{2 N} \frac{\partial^{2} \Psi_{N}(t, x)}{d t^{2}}=0
$$

and follow the prescription of Theorem 2 to obtain a variational principle equivalent to the equation (61), hence solving the Fourier equation in the impulse space and, due to the monotonicity of the exponential, reverse the expression for the action as

$$
S_{N}(t, x)=-\frac{1}{N} \ln \Psi_{N}(t, x)=-\frac{1}{N} \ln \sqrt{\frac{N}{2 \pi t}} \int d y \exp \left(N\left(S_{0}(y)+\frac{(x-y)^{2}}{2 t}\right)\right),
$$

that, in the thermodynamic limit, returns the solution as the inverse Legendre transform of the initial condition

$$
S(t, x)=\inf _{y}\left(\frac{(x-y)^{2}}{2 t}+S_{0}(y)\right)
$$

\section{Conclusions and Outlooks}

In this paper we have shown how to adapt techniques originally stemmed mainly in the classical mechanics scenario in order to make them powerful tools for solving the statistical mechanics of mean field spin systems too, focusing on bipartite structures in full interaction. In a sense this work extends, merges (and closes, at least at the replica symmetric level), our investigations started in [6] we and [7] on mean field spin systems in interaction. In particular, in this paper we considered the test case of two parties, each one provided of its internal links and in reciprocal interaction with the other party: we investigated both the ferromagnetic case, where parties share the positivity of the couplings (whose strength is instead tunable in each party and reciprocally) and the glassy counterpart, where, retaining the same freedom in the strengths, couplings are drawn at random from a Gaussian distribution allowing for positive and negative strengths, hence frustrating the network.

At first we proved that it is possible to built a sum rule for the free energy (strictly speaking the pressure) of these models in terms of a replica symmetric expression plus a rest that is exactly the source of order parameter fluctuations, then, if these order parameters are self-averaging (as in the ferromagnetic case or in the replica symmetric approximations), such an expression becomes the true solution in the thermodynamic limit. We stress that, however, for glassy systems, in a huge region of the tunable parameters (namely where the rest in the sum rule is positive defined) such an expression is further a rigorous bound for the real free energy.

If self-averaging is lacking, instead, as in the low temperature limit of glassy systems, the expression for the free energy is only an approximation. We remark however that in several applicative fields (e.g. ranging from neural to immune or metabolite networks in theoretical biology) this level of description is retained, hence motivating the present study. 
One step forward, we showed that there exists a sharp one to one mapping between the free energy of these systems in the statistical mechanics scenario and an action function in a suitably defined fictitious spacetime such that solving the latter implies solving the former: following this path, we have shown how to obtain an explicit expression (again at the replica symmetric level) for the action and then map back this finding in the original statistical mechanics framework reobtaining the same solutions (both for ferromagnets and for glasses) previously discovered.

Lastly, we have shown that the Cole-Hopf transform of the free energy obeys a diffusion-like equation that we solved via the standard route of Green propagator and convolution theorem in the impulse space and then we mapped it back in the original frame, re-obtaining once more the same thermodynamics.

As a final remark, we stress here that extensions of these techniques to a (finite in number) amount of different species (beyond the test-case of two groups investigated here) is straightforward.

Summarizing, we believe that, while the self-averaging scenario is completely understood, from multiple perspectives, and rules out further investigations on ferromagnets with multi-species, the phenomenon of replica symmetry breaking in multiple spin-glasses still deserves much more efforts for being tackled.

We plan to investigate its structure in the near future.

\section{Acknowledgements}

This work is supported by the FIRB grant RBFR08EKEV. Further we thank Sapienza Universita' di Roma, Istituto Nazionale di Fisica Nucleare (INFN) and Gruppo Nazionale per la Fisica Matematica (GNFM, INdAM) for partial support.

\section{References}

[1] E. Agliari, A. Barra, A. Galluzzi, F. Guerra, F. Moauro, Multitasking associative networks, Phys. Rev. Lett. 109, 268101, (2012).

[2] E. Agliari, A. Barra, F. Guerra, F. Moauro, A thermodynamic perspective of immune capabilities, J. Theor. Biol. 287, 48-63, (2011).

[3] D. Amit, Modeling Brain Function, Cambridge University Press, 1989.

[4] A. Barra, E. Agliari, A statistical mechanics approach to autopoietic immune networks, J. Stat. Mech. 07, 07004, (2010).

[5] A. Barra, G. Genovese, F. Guerra, D. Tantari, A Solvable Mean Field Model of a Gaussian Spin Glass, submitted to J. Phys. A (2013).

[6] A. Barra, G. genovese, F. Guerra, Equilibrium statistical mechanics of bipartite spin systems, J. Phys. A 44, 245002, (2012).

[7] A. Barra, G. Del Ferraro, D. Tantari, Mean field spin glasses treated with PDE techniques, E. Phys. J. B 86, 332, (2013).

[8] A. Barra, P. Contucci, E. Mingione, D. Tantari, Multi-species mean-Peld spin-glasses. Rigorous results, submitted to Annals H. Poincare' (2013).

[9] A. Barra, The Mean Field Ising Model trough Interpolating Techniques, J. Stat. Phys. 132, 604, (2008).

[10] A. Barra, G. Genovese, F. Guerra, The Replica Symmetric Approximation of the Analogical Neural Network, J. Stat. Phys. 140, 784-796, (2010).

[11] A. Barra, G. Genovese, F. Guerra, D. Tantari, How glassy are neural networks?, J. Stat. Mech. 07, 07009, (2012). 
[12] A. Barra, A. Di Biasio, F. Guerra, Replica symmetry breaking in mean-field spin glasses through the Hamilton-Jacobi technique, J. Stat. Mech. 09, 09006, (2010).

[13] A. Barra, P. Contucci, Toward a quantitative approach to migrants integration, EuroPhys. Lett. 89, 68001, (2010).

[14] A. Barra, P. Contucci, R. Sandell, C. Vernia, A statistical mechanics approach to migrant's integration, Submitted to Sc. Rep. Nature, (2013).

[15] W. Brock, S. Durlauf, Discrete choice with social interactions, Rev. Econ. St. 68, 2133, (2001).

[16] J.P. Bouchaud, M. Potters, Theory of Financial Risk and Derivative Pricing, Cambridge University Press, 2004.

[17] A.C.C. Coolen, R. Kuhen, P. Sollich, Theory of neural information processing systems, Oxford University Press, 2005.

[18] A. C. C. Coolen, The Mathematical theory of minority games - statistical mechanics of interacting agents, Oxford Univ. Press, 2005.

[19] P. Contucci, Stochastic Stability: a Review and Some Perspectives, J. Stat. Phys. 138, 543, (2010).

[20] S.N. Durlauf, How can statistical mechanics contribute to social science?, Proc. Natl. Acad. Sc. 96, 10582 , (1999).

[21] P.S. Dodds, R. Muhamad, D.J. Watts, An Experimental Study of Search in Global Social Networks, Science, 301, 5634, (2003).

[22] I. Gallo, P. Contucci, Bipartite Mean Field Spin Systems. Existence and Solution, Math. Phys. Elec. Jou. 14, 1-22, (2008).

[23] M. Enquist, S. Ghirlanda, Neural networks and animal behavior, Princeton Univ. Press., 2005.

[24] K Fisher, J. Hertz, Spin glasses, Cambridge Studies in Magnetism, 1993.

[25] S. Galam, Sociophysics, Springer Press, 2005.

[26] G. Genovese, A. Barra, A mechanical approach to mean Peld spin models, J. Math. Phys. 50, 365234, (2009).

[27] F. Guerra, About the overlap distribution in mean field spin glass models, Int. J. Mod. Phys. B 10, 16751684, (1996).

[28] F. Guerra, Sum rules for the free energy in the mean field spin glass model, Fields Institute Communications 30, Amer. Math. Soc. (2001).

[29] F. Guerra, Broken Replica Symmetry Bounds in the Mean Field Spin Glass Model, Comm. Math. Phys. 233, 1-12, (2003).

[30] F. Guerra, F.L. Toninelli, Central limit theorem for fluctuations in the high temperature region of the Sherrington-Kirkpatrick spin glass model, J. Math. Phys. 43, 6224-6237, (2002).

[31] J. A. Hertz, A. S. Krogh, R. G. Palmer, Introduction to the theory of neural computation, Elsevier Press, 1993.

[32] J.M. Kincaid, E.G.D. Cohen, Phase diagrams of liquid helium mixtures and metamagnets: experiment and mean field theory, Phys. Lett. C 22, 58-142, (1975).

[33] S. Kirkpatrick, D. Sherrington, Solvable model of a spin-glass, Phys. Rev. Lett. 35 1792, (1975). 
[34] P. Lax, Hyperbolic systems of Conservation Laws and the Mathematical Theory of Shock Waves, SIAM, (1973).

[35] C. Martelli, A. De Martino, E. Marinari, M. Marsili, I. Perez Castillo, Identifying essential genes in E. coli from a metabolic optimization principle, Proc. Natl. Acad. Sc. 106, 2607, (2009).

[36] M. Mezard, G. Parisi, M. Virasoro, Spin glass theory and beyond, World Scientific Publishing, 1987.

[37] T. Mora et al., Maximum entropy models for antibody diversity, Proc. Natl. Acad. Sc. 107, 12, 5405, (2010).

[38] G. Parisi, A simple model for the immune network, Proc. Nat. Acad. Sc. 87, 2412-2416, (1990).

[39] G. Parisi, Toward a mean field theory for spin glasses, Phys. Lett. A 73, 203, (1979).

[40] G. Parisi, A sequence of approximated solutions to the $S$-K model for spin glasses, J. Phys. A 13, 115, (1980).

[41] G. Parisi, The order parameter for spin glasses: a function on the interval 0 - 1, J. Phys. A 13, 1101, (1980).

[42] M. Talagrand, The Parisi Formula, Annals of Mathematics 163, 221-263, (2006).

[43] M. Talagrand, Spin glasses: a challenge for mathematicians. Cavity and Mean field models Springer Verlag, 2003. 